\title{
Investigation into Channel Angular Extrusion and The UREAD Energy Absorption Technique
}

\author{
R. Lupoi ${ }^{1, a}$ and F.H. Osman ${ }^{1, b}$ \\ ${ }^{1}$ Department of Mechanical Engineering, University of Bath, \\ Claverton Down, BA2 7AY, Bath, United Kingdom. \\ a R.Lupoi@bath.ac.uk, ${ }^{b}$ F.Osman@bath.ac.uk
}

\begin{abstract}
Channel Angular Extrusion(CAE) processes are characterised by imposing high level of strain into deformable materials using simple tools where the material is contained within a specific path. Tool stresses could be critical. The process is capable of reducing the thickness of flat sheets or slabs, and using Equal Channels, it could be used for material refinement where internal grain size changes to nano-scale structure by intense shearing. In this paper, the applications of CAE have been extended to include the construction of Universal Re-useable Energy Absorption Devices (UREAD) using equal channels. An investigation is carried out into the distribution of contact stresses at the tool/material interface. The model employs the slab method of analysis and splits the material inside the channels, which intersects at $90^{\circ}$, into two zones; one causes the deformation while the other remains rigid. Normal stresses that may develop at the contact surface between the deformable material and the tools are analysed for various cases of CAE and are compared with those obtained by the Finite Element Analysis. Experimental investigation on UREAD devices using lead are also presented where the energy absorbed reached a maximum of $160 \mathrm{~J}$ for $100 \mathrm{~mm}$ cross sectional area and $30 \mathrm{~mm}$ stroke. An experimental set up for measuring contact pressure in CAE/ECAE is introduced and measurements at different depth of the CAE entry channel are presented. The sensing mechanism is based upon the sensitive pressure pin technique.
\end{abstract}

Keywords: CAE, ECAE, energy absorption, UREAD, Stress analysis, contact stresses, pressure pin technique. 


\section{Nomenclature}

$\begin{array}{ll}v_{l} & \text { vertical velocity component } \\ v_{2} & \text { horizontal velocity component } \\ b & \text { width of the vertical channel } \\ h & \text { width of the horizontal channel } \\ D & \text { diameter } \\ s & \text { pin distance } \\ L & \text { billet length in the vertical channel } \\ p & \text { normal stress on vertical direction } \\ \sigma_{x} & \text { normal stress on horizontal direction } \\ S & \text { resistance force } \\ p_{a v e} & \text { average normal stress } \\ p_{r}, p_{l} & \text { average normal stresses on tool sides } \\ F & \text { forming force } \\ \tau_{k} & \text { material yield shear stress } \\ \mu & \text { coefficient of friction }\end{array}$

\section{1- Introduction}

Technological advances that involve material deformation provide an opening to various applications in engineering and domestic products. Elastic and plastic deformation forms the basis for energy transformation or absorption in many small and large industrial and domestic products. In design areas of engineering systems, such as those included in static structures like buildings and dynamic bodies that are most common in transports, it is fundamental to take into account severe structural overloading, which may develop from the happening of accidents and crashes. Some are natural such as earthquakes and others are due to loss of control. Therefore, personal and structural safety is certainly a very changing discipline, which attracted the interest of many researchers. Protective devices and techniques must be part of the structural integrity of the system design for them to be effective. Moderate and high energy absorption devices vary considerably in working principles and mechanisms but they must dampen the damaging component of forces and prohibit undesirable oscillation or co-lateral damage. When plastically deformable metallic dampers are used to dissipate energy they incorporate thin metal sheets, tubes, torsion bars[1] 
so that the rate of energy absorption is considered through controlled local deformation. However, competing disciplines employ other forms of energy absorption schemes. Some devices employ the dry friction principles to dissipate energy. The Sumitomo friction device [2] provides an example of this class. In this case, energy is absorbed through the development of resistance forces between some contacting internal parts. More complicated arrangements were studied and developed, such as Active Bracings Systems (ABS) [3], which are widely used to protect high buildings. Energy, in this case, is dissipated through hydraulic actuators, activated by a power system and controlled through a feedback sensor based system. Actuators response can be adapted to various applied loads and operational conditions. Most current energy absorption devices may suffer from one of the inherent disadvantages e.g. existence of rebound force, unpredictable behaviour during impact, lack of re-usability, complexity in design and lack of flexibility.

In this paper, a revolutionary technique to dissipate energy is presented, it is based upon a recently developed concept; Universal Re-usable Energy Absorption Devices (UREAD) [4]. Such devices are flexible, adaptable to various applications, and could be tailored to different energy absorption requirements. UREAD employs a material deformation mechanism through the principles of the Equal Channel Angular Extrusion (ECAE) process [5]. In such a process, a billet of a deformable material is forced to flow through intersecting channels. Although severe plastic deformation occurs, the geometry of the material being deformed remains unchanged. From a historical point of view, ECAE has been used for micro structural refinement through the development of severe local deformation by intense shearing. The grain size of the deforming material was shown to decrease with increasing the number of extrusion passes in ECAE [6,7]. As a consequence, the evolution of grain refinement was attributed to the considerable increase in the material yield strength and hence the high extrusion tool forces [8]. It is worth noting that the initial geometry and the final geometry are the same even after multiple processing. Rosochowski [9] exploited such 
characteristics and introduced ECAE with multi $90^{\circ}$ intersections. For successful operation the increase in tool forces must be absorbed by the ECAE die. An analytical procedure and experimental study of tool stresses and cavity stresses in channel angular extrusion are presented in this paper. Also, ECAE application is extended to define the UREAD energy devices, their characteristics and their behaviour.

\section{2- Analysis of Plane Strain Channel Angular Extrusion}

Plane strain Channel Angular Extrusion(CAE), in general, is an asymmetric process in which material in the form of plates or sheets is subjected to intensive shearing process through which the material strength is increased considerably. Reduction in thickness is not necessarily a controlling parameter because shearing occurs when the material passes through intersecting channels.

\section{1- 90 ${ }^{\circ}$ CAE Deformation Model}

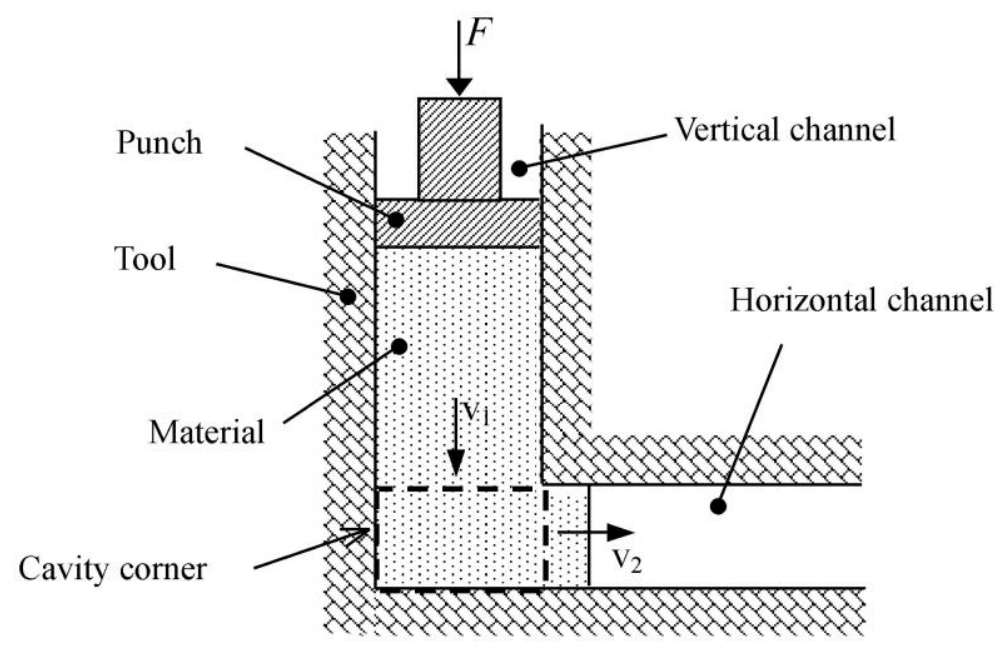

Figure 1. Schematic diagram of CAE Deformation mechanism

Fig. 1 shows a schematic diagram of the mechanism of deformation in the $90^{\circ} \mathrm{CAE}$ material forming processes. The process comprises two intersecting channels, a vertical and a 
horizontal channel in this case. The tools are simple and include a punch that forces a solid billet material to extrude from one channel to another through the application of a force $F$. The cavity corner encompasses the instantaneous local deformation by the change of the material velocity, it changes from $v_{1}$ in the vertical direction to $v_{2}$ in the horizontal direction.

\section{2- Stress analysis of cavity corner}

The geometrical properties and the proposed elemental deformation in a two-dimension $90^{\circ}$ CAE are shown in Fig. 2. The material is forced to deform from the vertical channel into the horizontal channel through widths $b$ and $h$ respectively. The length of the deformable material remaining in the vertical channel, $L$, is important when considering the force required to cause plastic deformation in the cavity corner.

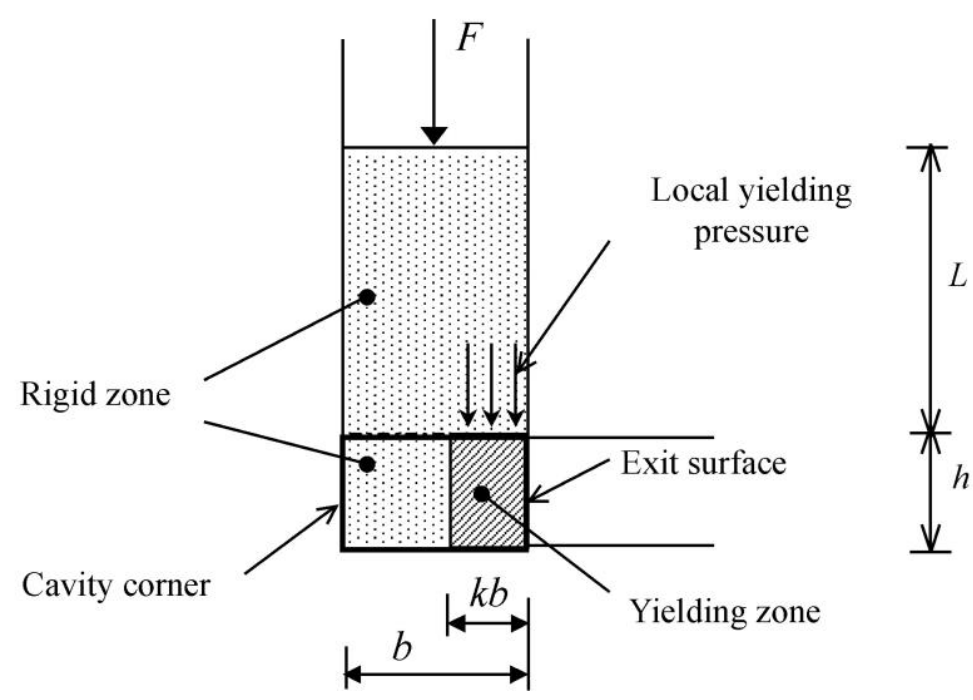

Figure 2. Stress-zone model in cavity corner

When yielding takes place, material flow will also depend on the opening in the horizontal channel, $h$. Also, additional hydrostatic pressure is required to overcome the elastic deformation of the surrounding tool forming the vertical channel, $L$. The hydrostatic pressure is at its minimal state in the open case of simple compression $(L=0)$ and maximum for enclosed cavity $(h=0)$. It is therefore proposed that at the instant of yielding, the deformation pattern consists of three zones, as shown in Fig. 2. Such zones are modelled to be separated 
by continuous lines, as represented in the Figure. A yielding zone seems to exist in the intersection volume between the two channels and begins from the exit surface. The remainder of the intersection volume stays rigid as well as the material in the vertical channel. The deformation of the yielding zone is effected by local yielding and does not extend immediately to include the cavity corner. A parameter, $k$, is now introduced which defines the width of the yielding zone in relation to the width of the vertical channel. Hence the width of the yielding zone is given by $k b$, where $k$ is between zero and one. Also, it is assumed that the yielding process in the intersection between the channels is affected by the static pressure in the stressed rigid material in the vertical channel. Accordingly, the following relationships are constructed for the cases where $h \leq b$;

$$
\begin{array}{ll}
k=\frac{L}{h} \text { for } & 0 \leq L<h \\
k=1 \text { for } & L \geq h
\end{array}
$$

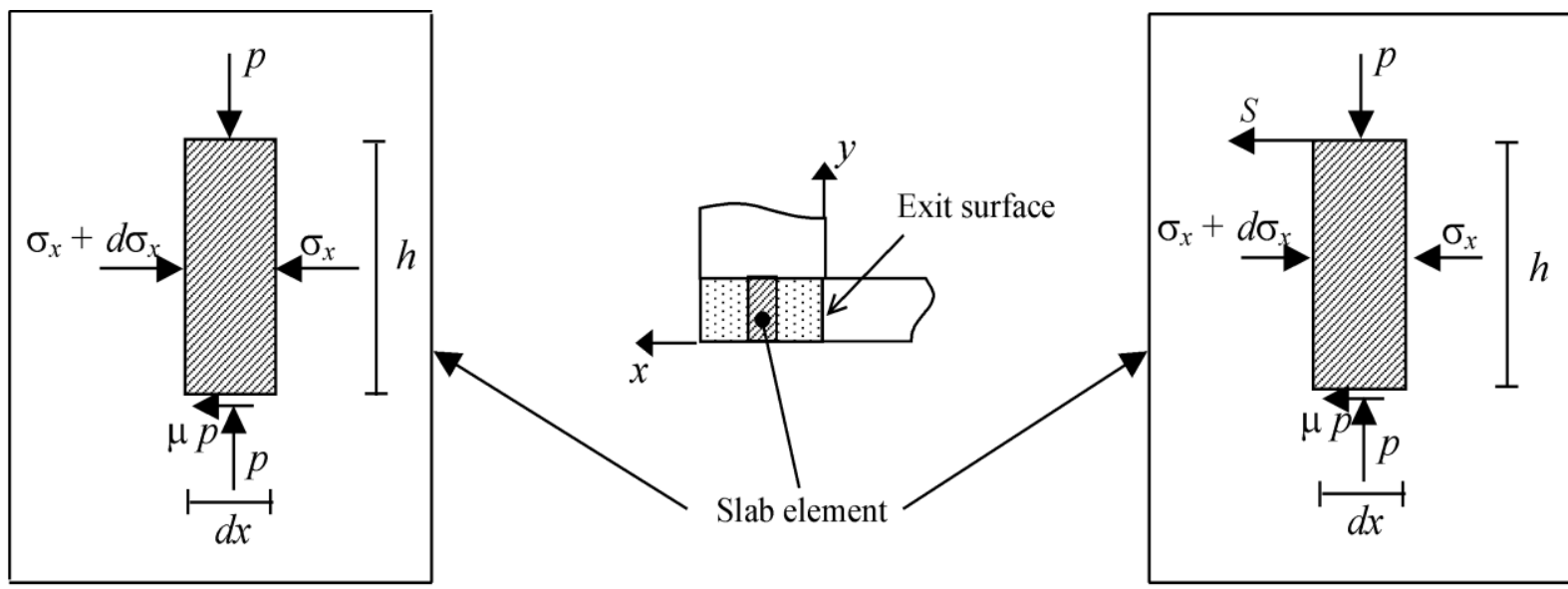

Rigid zone

Yielding zone

Figure 3. Stress system in cavity corner

Fig. 3 shows a slab element, $d x$, that is located in the cavity corner at a distance $x$ from the exit surface. This element may exist in the yielding zone or the rigid zone but within the cavity corner as shown in Fig. 3. The stress system consists of the horizontal normal stress components, $\sigma_{x}$, acting on the vertical surfaces, the vertical pressure, $p$, acting on the 
horizontal surfaces, frictional stress, $\mu$, acting along the interface between the tool and the deforming material, and a resistant force, $S$, that exists and acts on the interface between the rigid zone in the vertical channel and the yielding zone. However, the effect of $S$ on the rigid zone in the corner cavity would be negligible. Applying force equilibrium in the horizontal direction, $x$

$$
\begin{array}{ll}
-\left(\sigma_{x}+d \sigma_{x}\right) h+S+\mu p d x+\sigma_{x} h=0 & {[0 \leq x<k b]} \\
-\left(\sigma_{x}+d \sigma_{x}\right) h+\mu p d x+\sigma_{x} h=0 & {[k b \leq x \leq b]}
\end{array}
$$

Eq. 3 and Eq. 4 represent the stress distribution for the yielding zone and rigid zone respectively. The traditional assumptions also apply where the material is isotropic, homogeneous, incompressible, and its inertia has negligible effects on the stress system[10]. In the slab analysis [10], it is possible to assume that friction exists only at the boundary and has very little influence on the direction of the principal stresses. In addition, the material is constrained to flow between two parallel surfaces and therefore the principal stress directions in this case are horizontal and vertical inside the deforming volume of the yielding zone.

Applying Tresca yield condition, $\left(p-\sigma_{x}=2 \tau_{k}\right)$ where $\tau_{k}$ is the material yield shear stress gives;

$$
d \sigma_{x}=d p
$$

As shown in Fig. 3, the exit surface is a free surface and the vertical pressure, $p$, at the boundary between the yielding zone and the rigid zone is the same for both zones. The pulling force, $S$, is assumed to generate maximum sticking frictional conditions at the interface between the slab element and the rigid volume in the vertical channel and may be written as, $\tau_{k} d x$. Substituting Eq. 5 into Eq. 3 and Eq. 4 then integrating gives;

$$
\begin{array}{ll}
\frac{p}{2 \tau_{k}}=\frac{1}{2 \mu}(1+2 \mu) e^{\frac{x \mu}{h}}-\frac{1}{2 \mu} & {[0 \leq x<k b]} \\
\frac{p}{2 \tau_{k}}=\left[\frac{1}{2 \mu}(1+2 \mu) e^{\frac{k b \mu}{h}}-\frac{1}{2 \mu}\right] \cdot e^{\frac{\mu(x-k b)}{h}} & {[k b \leq x \leq b]}
\end{array}
$$


The average vertical pressure $p_{\text {ave }}$ acting on the intersection volume is obtained from Eq. 6 and Eq. 7, it is expressed in terms of the material yield stress as follows;

$$
\begin{aligned}
& \frac{p_{\text {ave }}}{2 \tau_{k}}=\frac{h}{b \mu}\left(\frac{1+2 \mu}{2 \mu}\right) \cdot\left[e^{\frac{b k \mu}{h}}-1\right]-\frac{k}{2 \mu}+\frac{\beta}{b} \cdot \frac{h}{\mu}\left[e^{\frac{\mu(b-k b)}{h}}-1\right] \\
& \text { where } \beta=\left[\frac{1}{2 \mu}(1+2 \mu) e^{\frac{k b \mu}{h}}-\frac{1}{2 \mu}\right]
\end{aligned}
$$

For the case of Equal Channel Angular Extrusion(ECAE), the width of the vertical channel, $b$, becomes equal to the width of the horizontal channel, $h$. Applying Eq. 6 and Eq. 7 to ECAE with $b=h=10 \mathrm{~mm}$ gives the results shown in Fig. 4. The coefficient of friction, $\mu$, is taken as 0.2 and the results are presented for different values of $L$ and $k$.

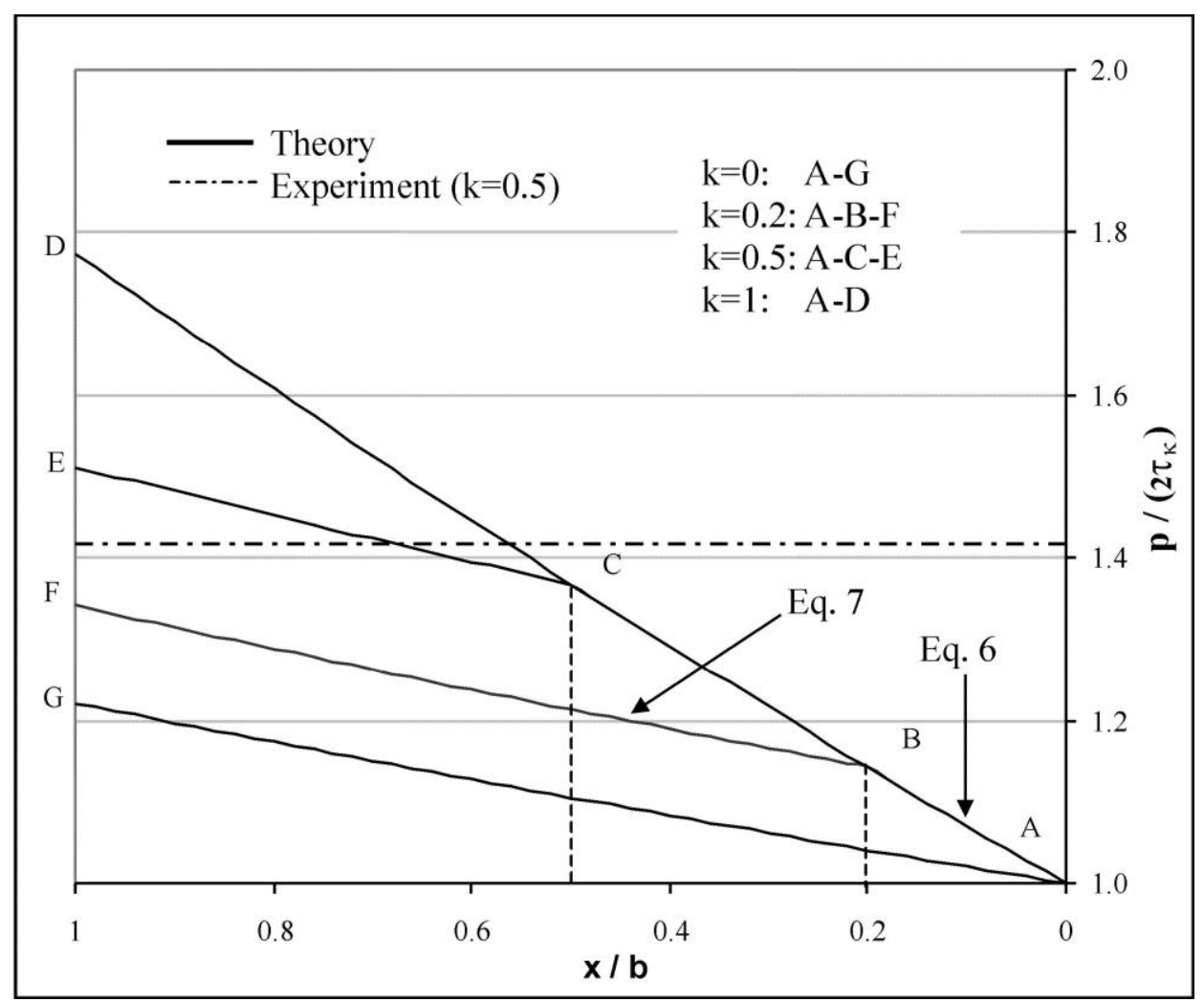

Figure 4. Vertical stress distribution in ECAE $(b=10, h=10)$ 
The distribution shows increasing stress values from the exit surface, $x=0$, to the cavity far side, $x=b$. Also, the stress level rises as the rigid volume in the vertical channel increases, until the limiting condition when $L$ is equal to $h$ or $k=1$. The initial yielding conditions in ECAE was performed experimentally on lead specimens using channels of $h=10 \mathrm{~mm}$ and $b=10 \mathrm{~mm}$ and acting displacement of $1 \mathrm{~mm}$. Billets were lubricated before each use and the material compressive yield stress was measured to $11 \mathrm{MPa}$. The billet rigid zone in the vertical channel, $L$, was $5 \mathrm{~mm}(k=0.5)$. The experimental average relative pressure is superimposed on the analytical results shown in Fig. 4. CAE with $b=10 \mathrm{~mm}$ and $h=5 \mathrm{~mm}$ is investigated analytically using Eq. 6 and Eq. 7, and distributions plotted in Fig.5 Comparison between the results of Fig. 5, ECAE, and those of Fig. 4, CAE, indicates significant increase in the acting pressure when the width of the exit channel is reduced by $50 \%$.

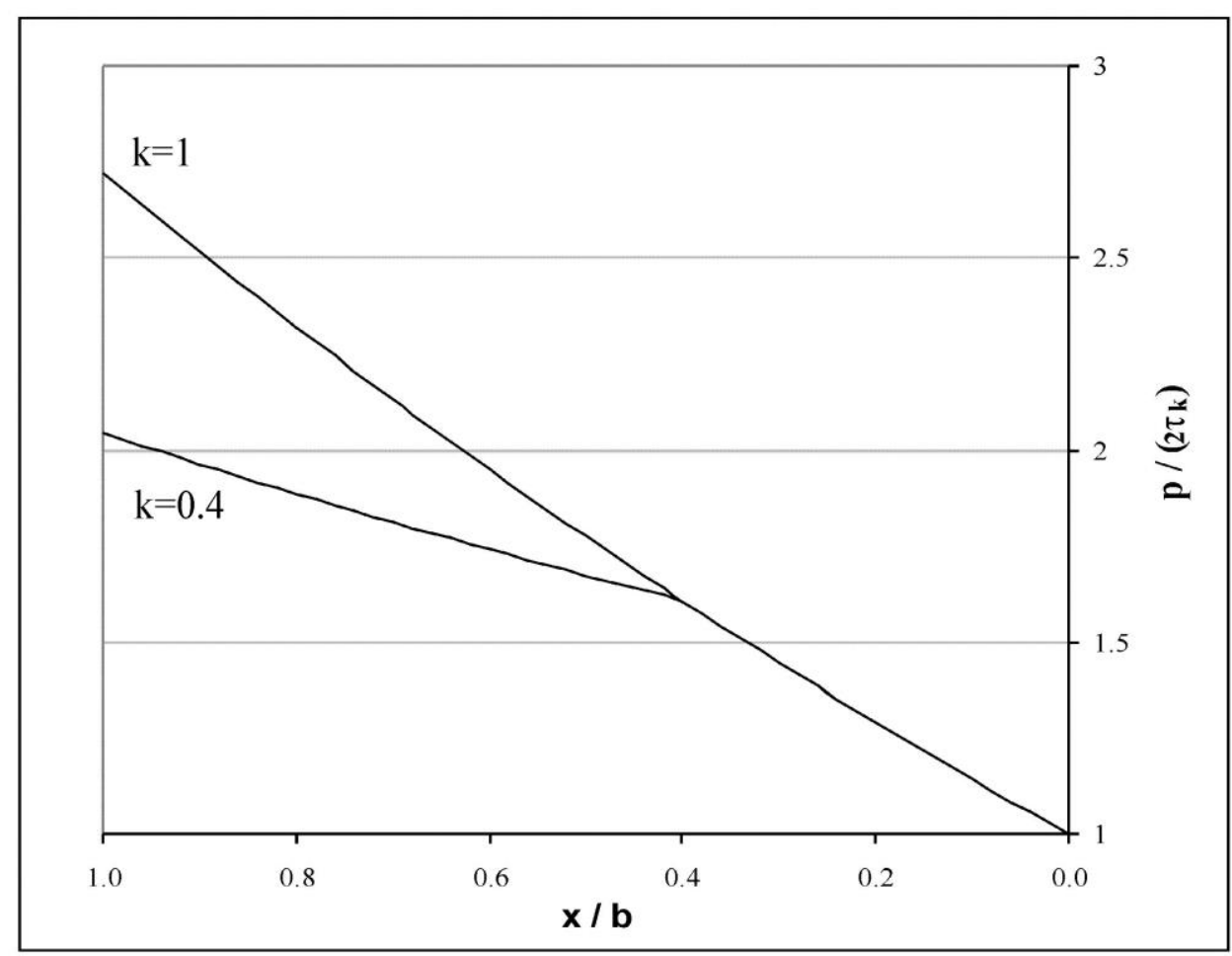

Figure 5. Theoretical vertical stress distribution in CAE $(b=10, h=5)$ 


\section{3- Stresses on the vertical channel}

The material in the vertical channel transmits the force to the yielding zone while remaining rigid, it is stressed from all directions. Fig. 6 shows the system of forces acting on all sides of this rigid zone, frictionless conditions are assumed on the sides of the vertical wall only. The average pressure, $p_{\text {ave }}$, given by Eq. 8 is superimposed as a hydrostatic component on all sides of the rigid zone. $\quad F_{r}$ and $F_{l}$ are the forces acting on the right and left side walls of the channel. $F_{t}$ is the effect of the material deforming in the cavity corner and exiting to the horizontal channel. However, $F_{t}$, is likely to be absorbed by the side wall that is in-line with the exit surface. The pressures $p_{r}$ and $p_{l}$ acting on the right and left side walls of the vertical channel may be written as;

$$
\begin{array}{lll}
p_{r}=p_{\text {ave }}+\frac{b}{h} \tau_{k} \text { and } \quad p_{l}=p_{\text {ave }} & {[0<L<h]} \\
p_{r}=p_{\text {ave }}+\frac{b}{L} \tau_{k} \quad \text { and } \quad p_{l}=p_{\text {ave }} & {[L \geq h]}
\end{array}
$$

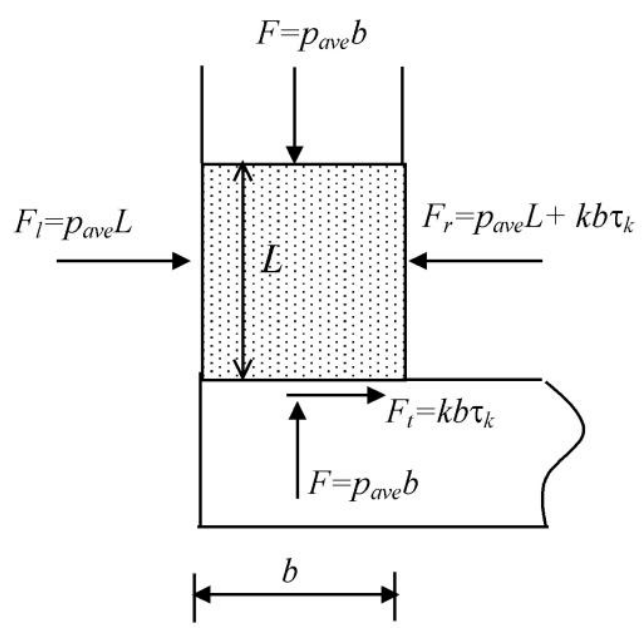

Figure 6. Force equilibrium on the vertical channel

Fig. 7 and Fig. 8 give the average side pressure on the left and right side walls of the vertical channel respectively. The results are given for different values of $L$ and the ratio $h / b$. The results suggest an increase in pressure on both sides of the wall for small values of $L$, until $L$ 
is comparable to $h$, after which the pressure remains constant on the left but decreases on the right side wall. For $L=0$ the pressure should instantly drop to zero.

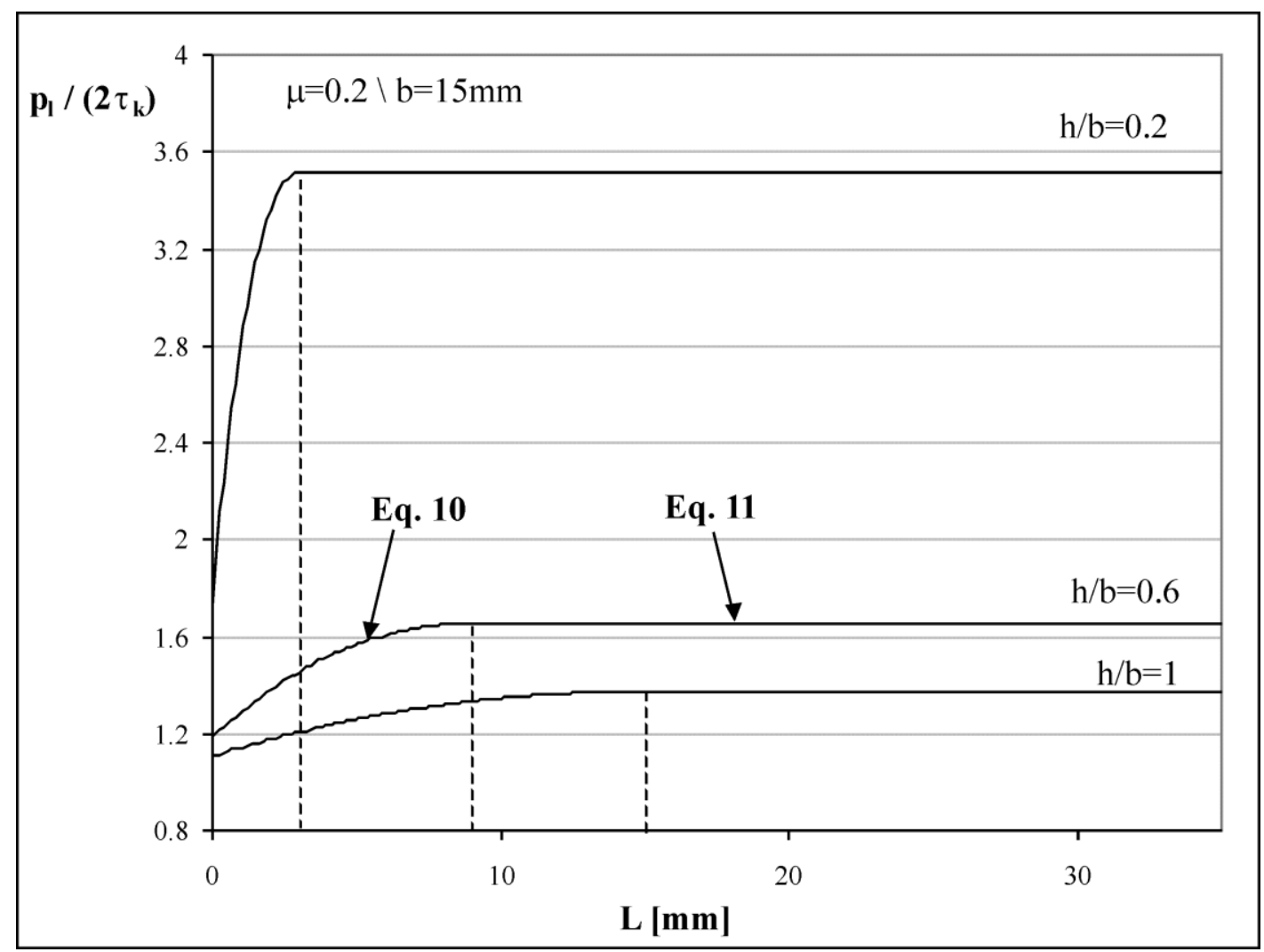

Figure 7. Pressure on the left side-wall of the vertical channel

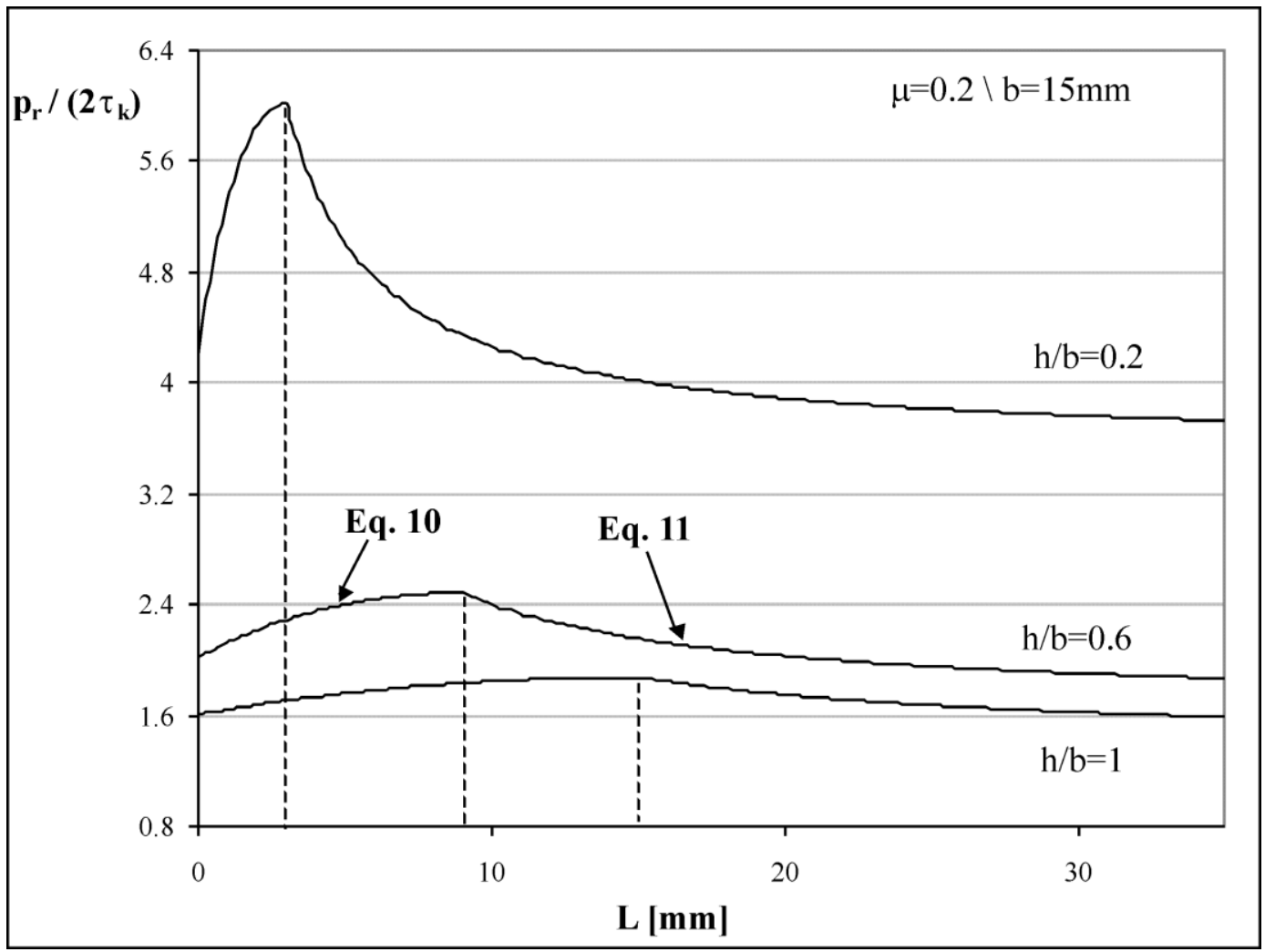

Figure 8. Pressure on the right side-wall of the vertical channel 


\section{3- Finite Element Simulation of Side and Internal Stresses}

The Finite Element Method was employed in order to investigate side tool and internal stresses in Channel Angular Extrusion(CAE). The analysis is performed by the commercially available software package ANSYS. The tool in this case was assumed rigid; therefore the tool boundaries were modelled by straight lines. The widths of the vertical and horizontal channels were $10 \mathrm{~mm}$ and $5 \mathrm{~mm}$ respectively with a constant friction coefficient of 0.2 at contact surfaces. The working material used in this analysis was to simulate lead. Fig. 9 shows the normal stress distribution on the tools at the initiation of yielding for plain strain CAE using different rigid billet length, $L$. The results appear to compare well with theoretical distribution shown in Fig. 7 and Fig.8. In the numerical analysis frictional forces on the sides of the vertical channel were taken into account, but not in the analytical solution, however, they do not seem to be of significance. Fig. 9 shows that the right hand side wall experiences higher stresses than that of the left hand side-wall. Also, it is noticed that the maximum predicted stresses are at the lower section of the right hand side-wall. Such distributions are also comparable to other numerical $[11,12]$ and theoretical investigations for the special case of ECAE [13], by employing different analysis techniques.

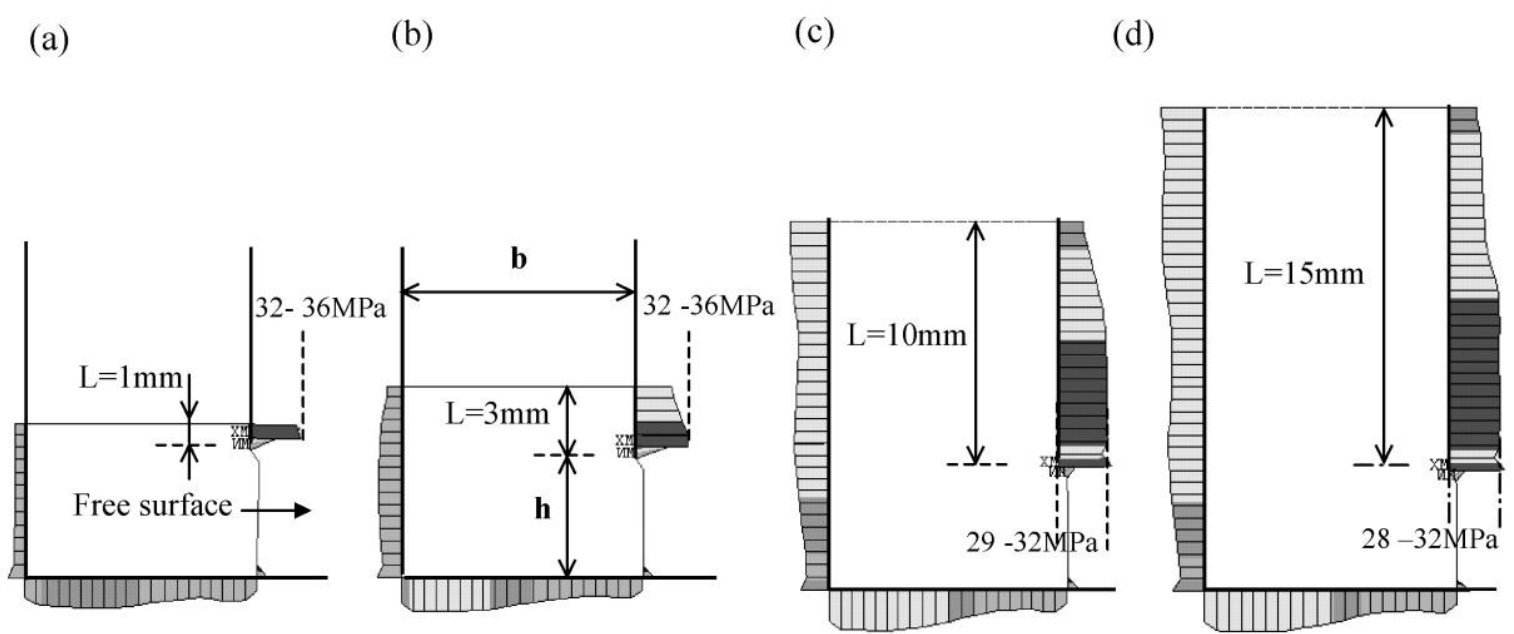

Figure 9. Tool sides normal stresses from a Finite Element Analysis 
Fig. 10 shows averaged values of side normal stresses numerical results on the left and right hand side wall, on a relative form. Results are plotted at various billet lengths in the vertical channel $(L=3,5,7,10 \mathrm{~mm})$. Specifically, the graph also includes a favorable theoretical comparison given by equations 10 and 11 . Both theoretical and numerical solutions seem to agree and be very close, however a higher difference gap is discovered for smaller billets lengths.

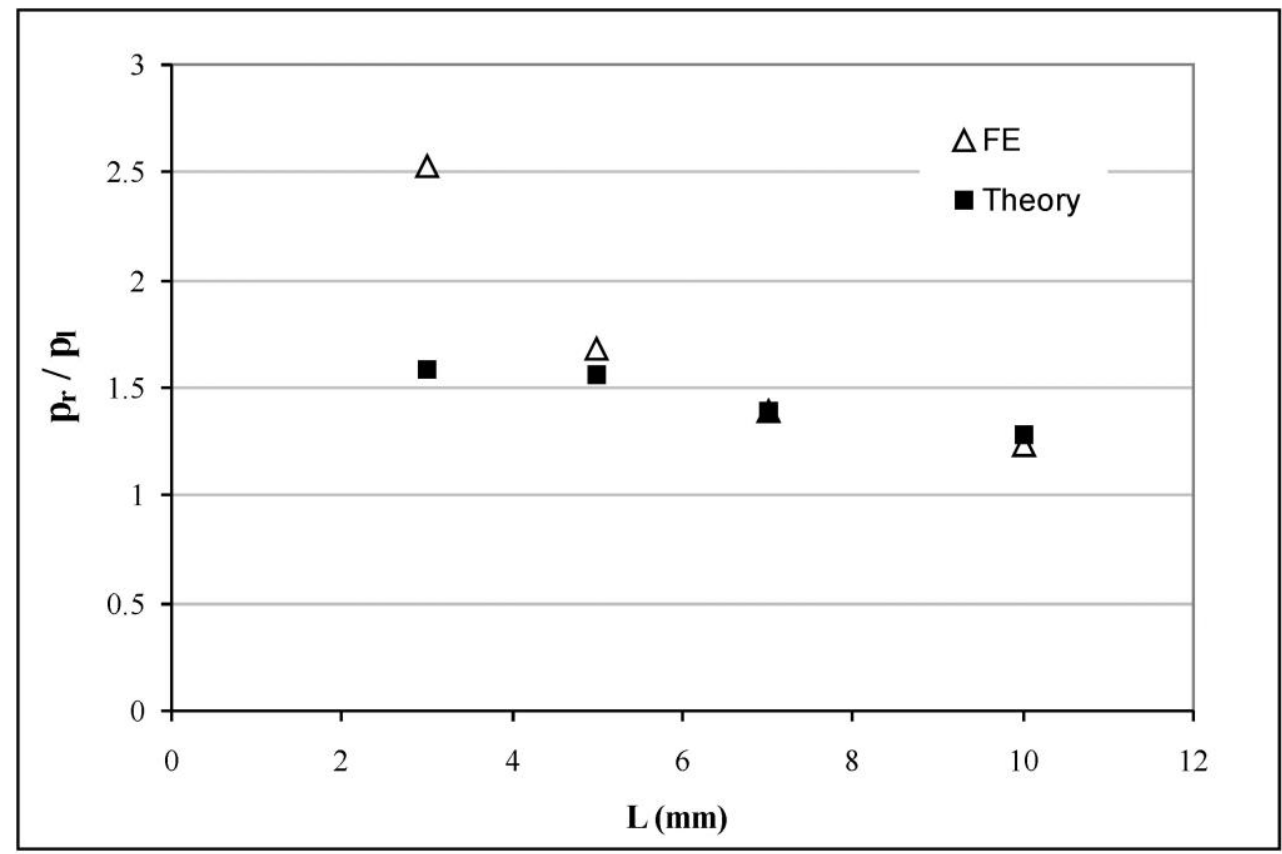

Figure 10. Numerical and theoretical relative normal stress on side tool walls $(b=10, h=5)$

Furthermore, Fig.11 compares the theoretical results showed in Fig.5, against numerical distributions by ANSYS, when $b=10$ and $h=5$. Numerical points seem to follow such analytical curves, showing agreement with the theoretical analysis. 


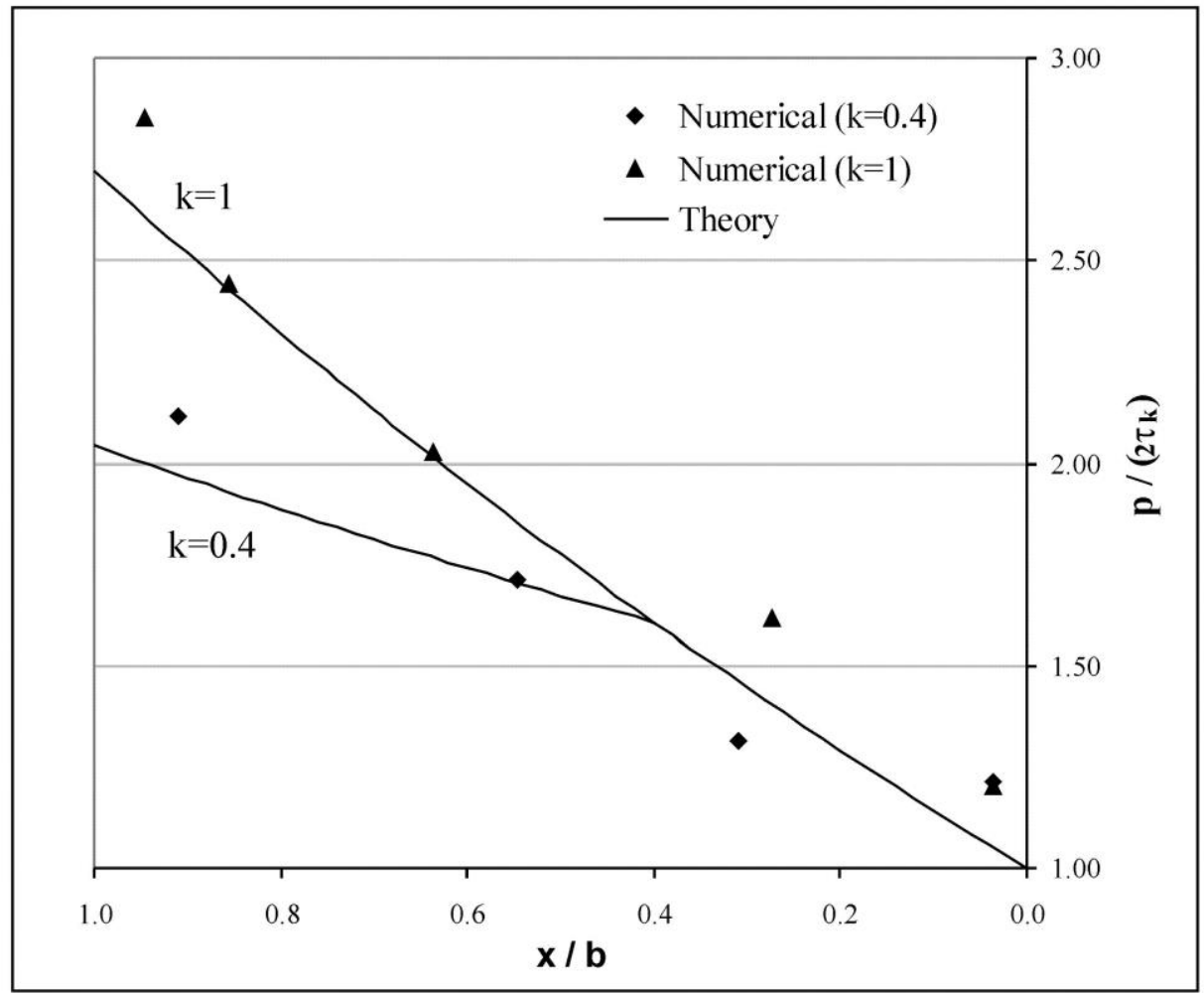

Figure 11. Numerical and theoretical vertical stress distribution in CAE $(b=10, h=5)$

\section{4- Universal Re-usable Energy Absorption Device - UREAD}

The CAE in its simplest form comprises the shearing of a deformable material at the intersection of channels of different or equal cross sectional area. The shearing process is an energy driven mechanism and therefore its use in energy absorption was introduced by Osman [4]. This represents a very new technology in that by exploiting the equality of channels cross sectional area in the ECAE, Osman developed Universal Re-usable Energy Absorption Devices (UREAD). Osman[4] described these devices as independent of the geometry of the channels cross sectional area as long as they form a passageway. However, simple rectangular and circular cross sectional area are easy to manufacture and serve the same purpose in respect to energy absorption. 


\section{1- Characterisation of Energy Channels}

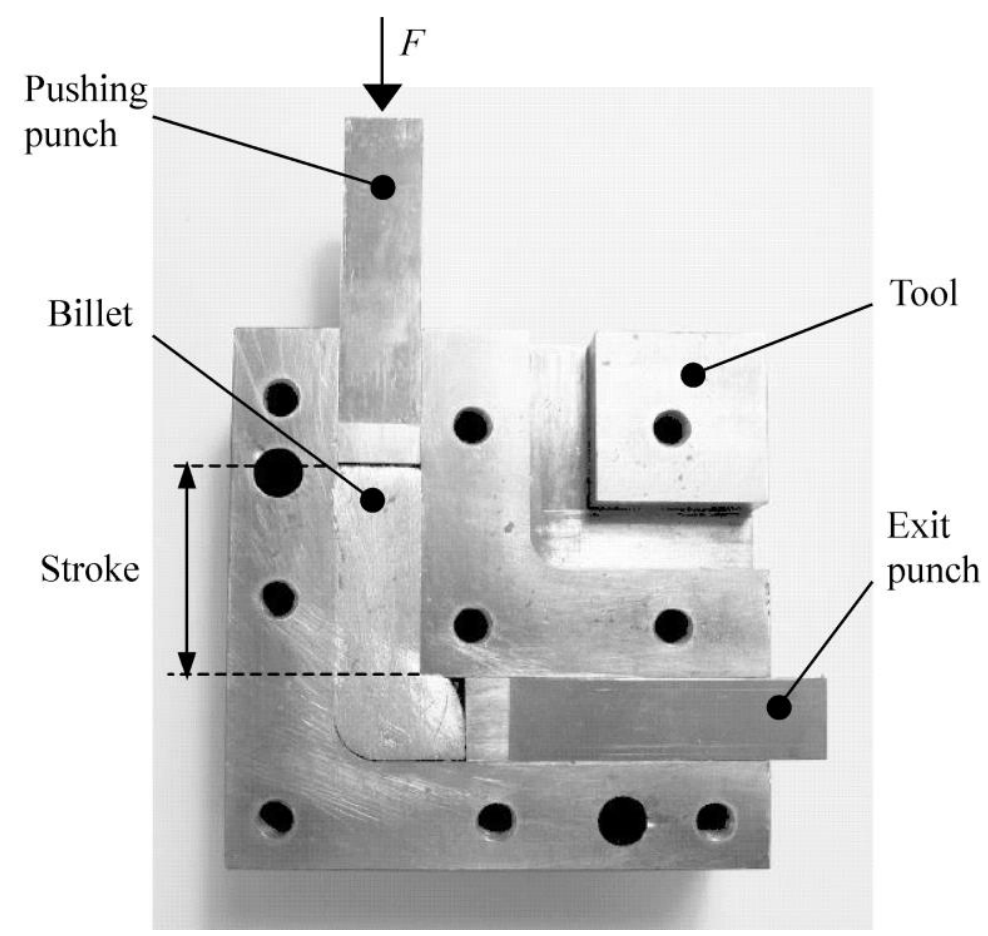

Figure 12. Typical design of a UREAD device

Fig. 12 illustrates a typical design of a device with rectangular cross sectional areas that form ECAE passageways with $90^{\circ}$ angle of intersection; two channels are used in this tool for research involving the characterisation of channel dimensions. From the same point of view, the tools were made out of H13 tool steel and heat-treated. In order to simulate the reusability of devices made of such passageways, two equal punches with equal maximum displacement are used in this experimental investigation. It is clear that for this design, the material can be pushed forward and backwards through the two channels several times. Therefore, the technology, as implemented, has the potential of infinite reusability. Various energy dissipation levels can be also achieved by the use of different deformable materials, such as copper and aluminium for high ranges, or lead, polymer and gums for medium/low ranges. Fig. 13 shows the experimental total energy dissipation characteristics of the UREAD devices shown in Fig. 12. Two rectangular channels $10 x 10 \mathrm{~mm}$ and 8x8mm, both 
with $90^{\circ}$ angle of intersection, were used with lead as the deformable material. Single stroke was employed in both cases; $30 \mathrm{~mm}$ and $17 \mathrm{~mm}$ for the large and small area respectively.

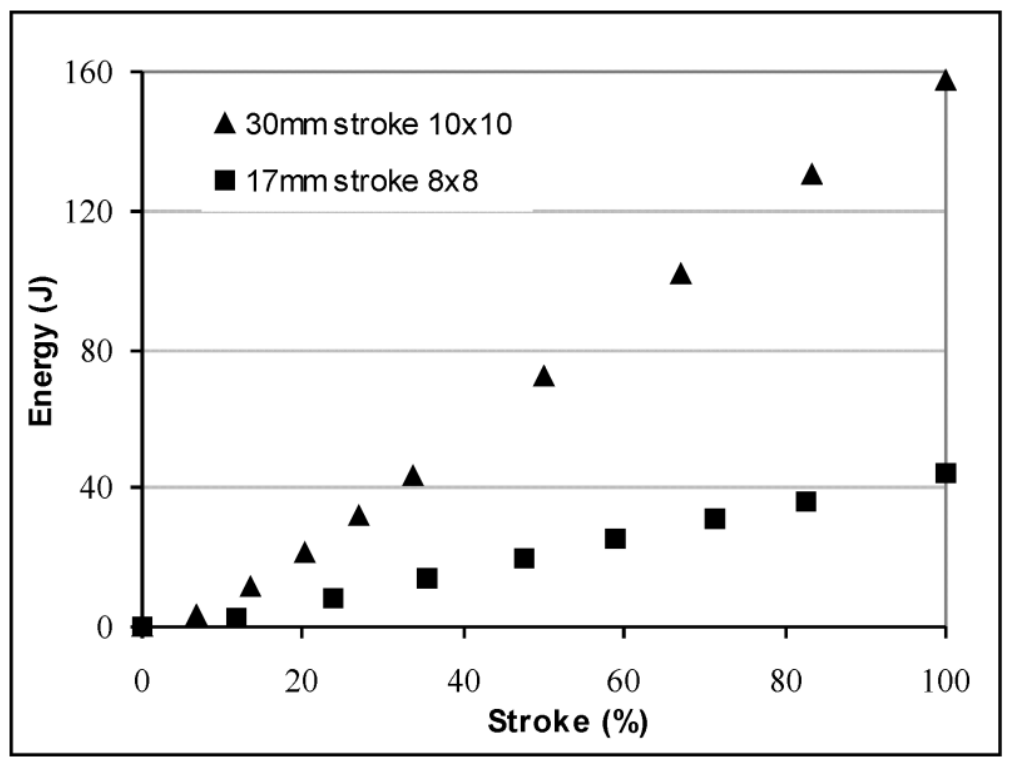

Figure 13. UREAD - $90^{\circ}$ channels experimental energy dissipation with lead

The energy requirements were obtain from the applied force, $F$, and punch displacement, which were recorded using a load cell, a linear potentiometer and a computer based data acquisition system. The results show a nearly linear behaviour with respect to the total energy absorbed in both channels, with higher energy levels from the channel with the larger cross sectional area.

\section{2- Experimental Measurements of Contact Pressure}

In order to provide some characteristics to the body of the UREAD device, knowledge of the internal stresses that it may experience is essential. Verification of analytical and numerical predictions of wall pressure in CAE and ECAE is not complete without some accurate experimental measurements, as difficult as this may be. The sensitive pressure pin technique is an effective method for the measurements of high tool stresses without damaging the measuring device. It consists of a small pin to be inserted into the tool to the point of 
interest, it is backed by a load cell that measures the local loading at that particular point[14]. A variation of this technique using a concealed under surface pressure pin was employed by Lupoi and Osman [15] where the pressure pin is kept away from the severe contact conditions.

\section{3- Tooling with Pressure Pin Construction}

A tool was designed for measuring the side pressure in UREAD- $90^{\circ}$ channels, it consists of three parts; the part of the tool that holds the CAE channel, a sensitive strain-gauged pressure pin and a pressure pin support as shown in Fig. 14. The tool was made out of H13 tool steel, where two channels, vertical and horizontal, inclined at $90^{\circ}$ were machined. The channel in this case had a circular cross sectional area with a diameter $D=15 \mathrm{~mm}$. Also, holes were machined on the side of the tool to accommodate the positioning of the pressure pin head, $2 \mathrm{~mm}$ in diameter, $1 \mathrm{~mm}$ away from the tool/material interface. The point where the pressure was measured was located at a distance $s$ from the bottom of the horizontal channel. Twopin system was used in this experiment.
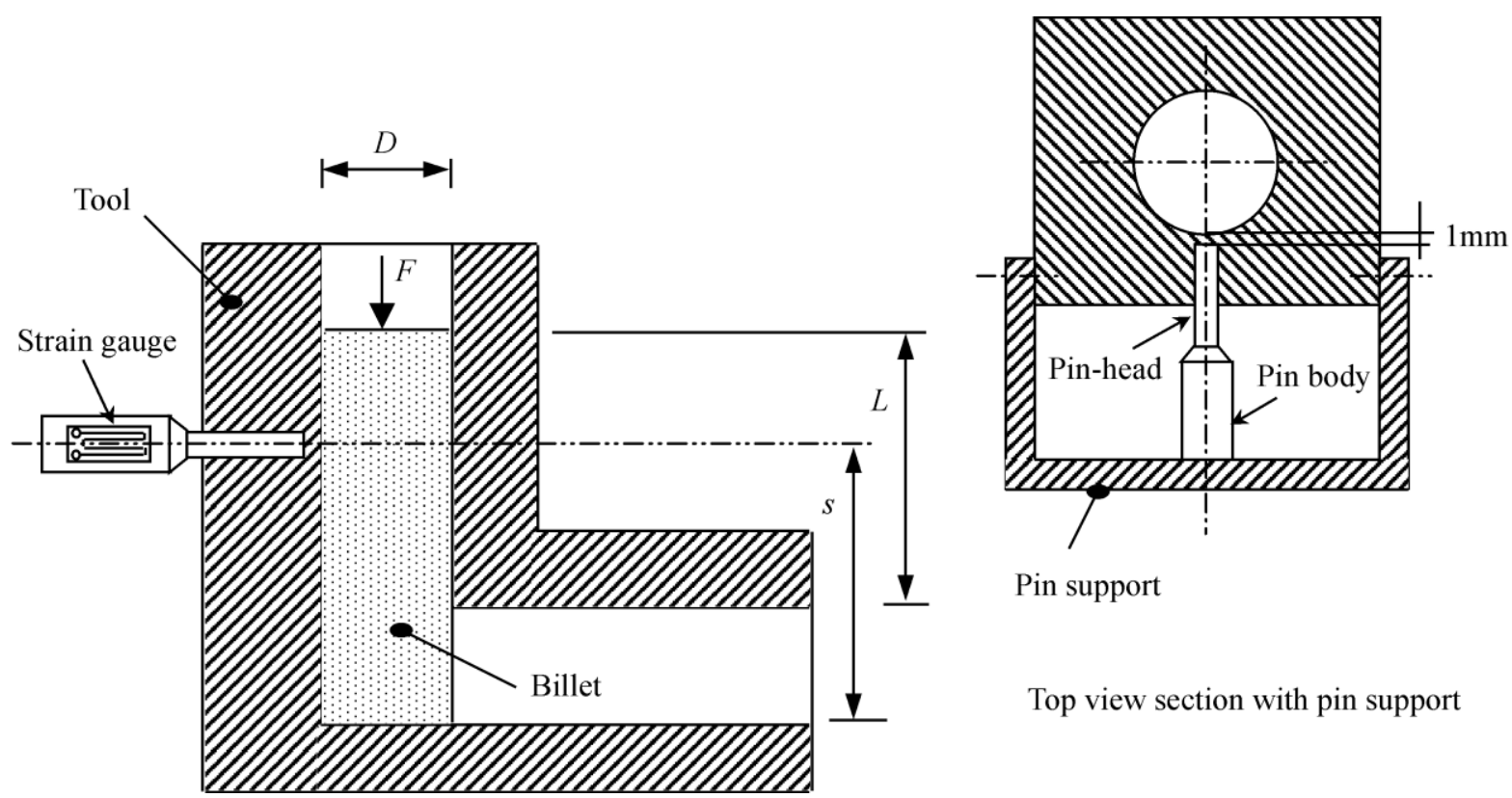

$$
\text { Pin support }
$$

Top view section with pin support 


\section{1- Experimental results of side Stresses}

A cylindrical billet of lead, of an initial length $L+D=41.5 \mathrm{~mm}$, was well lubricated and positioned in the vertical channel. The experimental set up was equipped with a multichannel data acquisition system. The pins were calibrated against known loads then a full stroke was carried out where the billet was forced to flow through the horizontal channel. Signals were recorded for each pressure pin throughout the punch stroke. The two pins, known as Pin1 and Pin2, were located at distances of $s=26$ and $11 \mathrm{~mm}$ respectively.

Fig. 15 gives the relative pressure measurement between Pin1 and Pin2 in the range of stroke where both pins were active. It also shows that the ratio between the pressure at the top of the vertical channel and that near the corner cavity is higher at the beginning of stroke than at the end of the stroke.

Such experimental results can be also favorably compared against numerical estimation of CAE side pressure, shown in Fig. 9. In fact, the side pressure level on the left hand side wall seems to increase from lower positions, near the cavity corner, to upper positions next to the top-surface of the billet, especially for relatively long billets in the vertical channel, as Fig. 9 (d) shows.

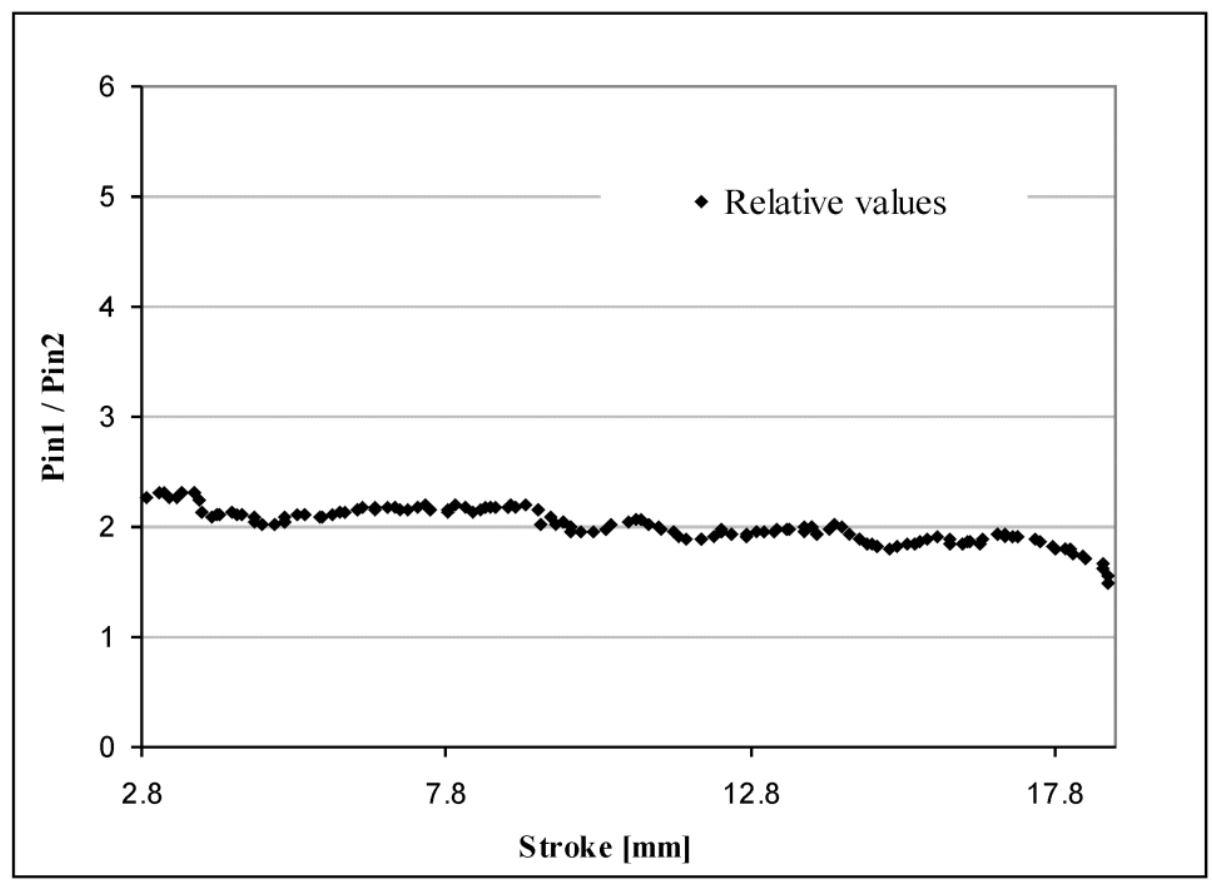

Figure 15. Relative measurements on the sidewall of the tool at different positions 


\section{5- Conclusions}

The Channel Angular Extrusion has been investigated analytically and numerically. The analytical solution gave prediction of tool stresses in such processes comparable to the numerical solution. Initial billet length in the vertical channel and channels geometry were identified as critical parameters for the starting of the yielding process and the develop of side-wall stresses.

The deformation pattern of the special case of Equal Channels Angular Extrusion (ECAE) was used as an energy absorption mechanism. The energy is dissipated through intense shearing while the level of energy dissipated depends on the material and cross sectional area of the channel as demonstrated by experiments. The characteristics of the process where a piece of material deforms without change in geometry was shown to potentially produce Universal Re-usable Energy absorption devices.

Experimental investigation into the measurements of tool stress and using the sensitive pressure pin technique showed that during the process the wall pressure distribution in the pressing channel assumes lower values at locations nearby the cavity corner.

\section{References}

[1] Juan Enrique Martinez -Rueda, "On the Evolution of Energy Dissipation Devices for Seismic Design”, Earthquake Spectra, 18 (2002), 2, 309-346.

[2] T.T Soong, G.F Dargush, "Passive energy dissipation systems in structural engineering", Wiley, 1996.

[3] M. Reinhorn, T.T. Soong “Active bracing systems”, Modern Steel Construction, 33 (1993), 28-33.

[4] Fayek Osman, "Reusable Energy Dissipation Device", Patent, W0 2004/044450 A1, 2004. 
[5] V.M. Segal, "Materials processing by simple shear", Materials Science and Engineering A, 197 (1995), 157-164.

[6] V.M. Segal, K.T. Hartwig, R.E. Goforth, "In situ composites processed by simple shear", Materials Science and Engineering A, 224 (1997), 107-115.

[7] V.M. Segal, "Equal channel angular extrusion: from macromechanics to structure formation", Materials Science and Engineering A, 271 (1999), 322-333.

[8] Zubear Ahmed Khan, Uday Chakkingal, P. Venugopal, "Analysis of forming loads, microstructure, development and mechanical property evolution during equal channel angular extrusion of a commercial grade aluminum alloy", Jour. of Mat. Proc. Tech., 135 (2003), 59-67.

[9] A. Rosochowski, L.Olejnik, M.Richert, "3D-ECAP of square aluminum billets”, proc. of the $8^{\text {th }}$ ESAFORM conference, (2005).

[10] R. Hill, "The mathematical theory of plasticity", Clarendon Press, 1985.

[11] C.J. Luis, R.Luri, J.Leon, "Strain and temperature analysis of AA-1370 processed by ECAE at different temperatures", Jour. of Mat. Proc. Tech., 164-165 (2005), 15301536.

[12] A. Rosochowski, L.Olejnik, "Numerical and physical modelling of plastic deformation in 2-turn equal channel angular extrusion", Jour. of Mat. Proc. Tech., 125-126 (2002), 309316.

[13] V.M. Segal,"Slip line solutions, deformation mode and loading history during equal channel angualr extrusion“, Materials Science and Engineering A, 345(2003), 36-46.

[14] F.H. Osman, F. Potel, M.S. Loveday, "Three-dimension analysis of contact stress measurements in forming processes", Proc. $6^{\text {th }}$ Int. Conf. on Technology of Plasticity(ICTP), Nuremberg, Germany, September, 1999, 391-396

[15] R.Lupoi and F.H.Osman, “ Under surface pressure sensing technique for the evaluation of contact stresses", Jour. of Mat. Proc. Tech, 164-165 (2005), 1537-1543. 